\title{
THE TRANSFORMATION OF SERIES AND SEQUENCES
}

\author{
BY \\ W. T. SCOTT AND H. S. WALL
}

In this paper we present some results on certain aspects of the theory of summation of series and sequences. The paper is divided into three parts:

I. Linear manifolds of Hausdorff means.

II. Gronwall summability.

III. A method of summation arising from an algorithm of Schur.

The principal results of each part are summarized in the introductory paragraph of that part.

\section{Linear Manifolds of HaUSdorfF means}

1. Introduction to Part I. The $n$th Hausdorff mean [7](1) of a series $\sum_{p=0}^{\infty} u_{p}$ is defined by

$$
U_{n}=\sum_{p=0}^{n} C_{n, p} \Delta^{n-p} c_{p} \cdot\left(u_{0}+u_{1}+\cdots+u_{p}\right), \quad n=0,1,2, \cdots,
$$

where $C_{n, p}=n ! / p !(n-p) !, \Delta^{i} c_{j}=c_{j}-C_{i, 1} c_{j+1}+C_{i, 2} c_{j+2}-\cdots$, and $\left\{c_{p}\right\}$ is a moment sequence, i.e.,

$$
c_{p}=\int_{0}^{1} u^{p} d \phi(u), \quad p=0,1,2, \cdots, \phi(u) \in B V[0,1]\left({ }^{2}\right) .
$$

The method of summation which assigns to the series $\sum u_{p}$ the sum $s$ when $U_{n} \rightarrow s$ will be denoted by $[H, \phi(u)]$ or by $\left[H, c_{p}\right]$, and called a Hausdorff mean. $[I I, \phi(u)]$ is regular $\left({ }^{3}\right)$ if and only if $\phi(u)$ is continuous at $u=0$ and $\phi(1)-\phi(0)=1$. We shall say that $[H, \phi(u)]$ is essentially regular if $\phi(u)$ is continuous at $u=0$. In this case, if $\phi(1)-\phi(0)=c_{0} \neq 0$, the mean $\left[H, \phi(u) / c_{0}\right]$ is regular.

It will be observed that if in (1.2) the integrand $u^{p}$ is replaced by some other suitably chosen function, e.g., $u^{p+1}$, then no restriction need be placed upon $\phi(u) \in B V[0,1]$ in order that the resulting mean be essentially regular.

Presented to the Society in three parts: Linear manifolds of Hausdorff means, November 22, 1940; Gronwall summability, April 12,1941; The transformation of series and sequences, April 12, 1941; received by the editors January 23, 1941, and, in revised form, February 28, 1941. We are grateful to Professor Einar Hille for directing our attention to the possibility of connecting our investigations with the ideas of Gronwall.

(1) Numbers in square brackets refer to bibliography at end of paper.

(2) $B V[a, b]$ is the class of all functions, real and complex, of a real variable $u$, which are of bounded variation on $a \leqq u \leqq b$.

(3) That is, it assigns to any convergent series $\sum u_{n}$ the value $\sum u_{n}$. 
In the light of this observation, we consider the problem of determining conditions upon a sequence of functions $\left\{\beta_{p}(u)\right\}$ in order that ( $\left.{ }^{4}\right)$

$$
c_{p}=\int_{0}^{1} \beta_{p}(u) d \phi(u), \quad p=0,1,2, \cdots,
$$

be a moment sequence for every $\phi(u)$ in $B V[0,1]$; and of determining further conditions under which $\left[H, c_{p}\right]$ is essentially regular. The results are contained in Theorems 2.1-2.4. We call the set of all means obtained with a given sequence $\left\{\beta_{p}(u)\right\}$ a (linear) manifold, denote it by $M\left[\beta_{p}(u)\right]$, and call $\left\{\beta_{p}(u)\right\}$ the basis of the manifold.

Perhaps the chief interest in this theory lies in the fact that we are able to obtain infinite classes of means all including a given mean or all equivalent to a given mean. For instance, if in $(1.3) \beta_{p}(u)=1 /(1+p u)$ and $\phi(u)$ is monotone non-decreasing, $\phi(1)-\phi(0)=1$, then $\left[H, c_{p}\right]$ is a regular mean included in $(C, 1)$; is equivalent to $(C, 1)$ if and only if $\int_{0}^{1} d \phi(u) / u$ converges; and is equivalent to $(C, 0)$ (convergence) if and only if $\phi(u)$ is discontinuous at $u=1$.

2. Conditions for a basis. One readily sees that necessary conditions upon a sequence of functions $\left\{\beta_{p}(u)\right\}$ in order that it be a basis for a manifold $M\left[\beta_{p}(u)\right]$ are:

(a) $\beta_{p}(u)$ is continuous for $0 \leqq u \leqq 1$;

(b) $\left\{\beta_{p}(u)\right\}$ is a moment sequence for each fixed $u, 0 \leqq u \leqq 1$.

The first of these conditions is necessary in order that the Stieltjes integral $\int_{0}^{1} \beta_{p}(u) d \phi(u)$ exist for all $\phi(u)$ in $B V[0,1]$. The second is necessary in order that the sequence of these integrals be a moment sequence when $\phi(u)$ is a step function with a single point of increase. From (b) it follows that there must exist a function $\left(^{5}\right) B(u, t)$ of bounded variation in $t$ for each $u, 0 \leqq u \leqq 1$, such that

$$
\beta_{n}(u)=\int_{0}^{1} t^{n} d_{t} B(u, t), \quad n=0,1,2, \cdots
$$

If (a), (b) hold, then a sufficient condition for $\left\{\beta_{p}(u)\right\}$ to be a basis is that

$$
\sum_{p=0}^{n} C_{n, p}\left|\Delta^{n-p} \beta_{p}(u)\right|<M,
$$
$M$ independent of $n, u$.

In fact, if

$$
a_{n}=\int_{0}^{1} \beta_{n}(u) d \alpha(u), \quad \alpha(u) \in B V[0,1]
$$

then, when (2.2) holds,

(4) Throughout this paper the integrals are in the Riemann-Stieltjes sense.

(5) This function is uniquely determined to an additive constant for all $t$ where it is continuous [7]. 


$$
\sum_{p=0}^{n} C_{n, p}\left|\Delta^{n-p} a_{p}\right|<M V,
$$

where $V$ is the total variation of $\alpha(u)$ on the interval $0 \leqq u \leqq 1$, and consequently $\left\{a_{p}\right\}$ is a moment sequence by virtue of a theorem of Hausdorff [7]. Condition (2.2) is met, in particular, if $\left\{\beta_{p}(u)\right\}$ is a totally monotone sequence $\left({ }^{6}\right)$ for each $u, 0 \leqq u \leqq 1$. In this case we shall call $\left\{\beta_{p}(u)\right\}$ a totally monotone basis.

THEOREM 2.1. If $B(u, t)$ is real and of bounded variation in $t, 0 \leqq t \leqq 1$, uniformly for all $u$ in the interval $0 \leqq u \leqq 1$, and if the functions $\beta_{n}(u)$ given by (2.1) are continuous functions of $u, 0 \leqq u \leqq 1$, then $\left\{\beta_{p}(u)\right\}$ is a basis for a manifold $M\left[\beta_{p}(u)\right]$.

REMARK. A sufficient condition [3] for the continuity of the functions $\beta_{p}(u)$ is that $B(u, t)$ be of bounded variation in $t$ uniformly for all $u$, and be continuous in $u$ for an everywhere dense set of values of $t$ including $t=0,1$. This everywhere dense set may depend upon $u$.

To prove the theorem, put $P(u, t)=\frac{1}{2}\left(\int_{0}^{t}\left|d_{t} B(u, t)\right|+\int_{0}^{t} d_{t} B(u, t)\right)$, $N(u, t)=\frac{1}{2}\left(\int_{0}^{t}\left|d_{t} B(u, t)\right|-\int_{0}^{t} d_{t} B(u, t)\right)$. It is no restriction to assume that $B(u, 0)=0$. Then $B(u, t)=P(u, t)-N(u, t)$, and $P(u, t), N(u, t)$ are monotone non-decreasing functions of $t$ for each fixed $u$. We then have

$$
\begin{aligned}
S_{n}=\sum_{p=0}^{n} C_{n, p}\left|\Delta^{n-p} \beta_{p}(u)\right| & =\sum_{p=0}^{n} C_{n, p}\left|\Delta^{n-p} \int_{0}^{1} t^{p} d_{t} B(u, t)\right| \\
& \leqq \sum_{p=0}^{n} C_{n, p}\left|\Delta^{n-p} \pi_{p}(u)-\Delta^{n-p_{\nu}}(u)\right|,
\end{aligned}
$$

where $\pi_{p}(u)=\int_{0}^{1} t^{p} d_{t} P(u, t), \nu_{p}(u)=\int_{0}^{1} t^{p} d_{t} N(u, t)$. Hence,

or

$$
S_{n} \leqq \sum_{p=0}^{n} C_{n, p} \Delta^{n-p} \pi_{p}(u)+\sum_{p=0}^{n} C_{n, p} \Delta^{n-p_{\nu}}(u)
$$

$$
S_{n} \leqq \pi_{0}(u)+\nu_{0}(u)=T(u),
$$

where $T(u)$ is the total variation of $B(u, t)$ in the interval $0 \leqq t \leqq 1$. Since, by hypothesis, $T(u)<M$, where $M$ is independent of $u$, we see that (2.2) holds, and the theorem is thereby established.

Definition. A manifold is called regular if it contains at least one regular mean, and if every mean contained in it is essentially regular.

TheOREM 2.2. A totally monotone basis $\left\{\beta_{p}(u)\right\}$ is the basis of a regular manifold if and only if $\beta_{0}(u) \not \equiv 0$, and

(6) A real sequence $\left\{c_{n}\right\}$ is totally monotone if all differences $\Delta^{m} c_{n} \geqq 0$. 


$$
\lim _{n=\infty} \Delta^{n} \beta_{0}(u)=0,
$$

Proof. The condition $\beta_{0}(u) \not \equiv 0$ is obviously necessary. Let $\left[H, a_{p}\right]$ be any mean in $M\left[\beta_{p}(u)\right]$. Then $\left[H, a_{p}\right]$ is essentially regular if and only if [7] $\lim _{n=\infty} \Delta^{n} a_{0}=0$. It readily follows that the condition (2.4) is necessary in order that $M\left[\beta_{p}(u)\right]$ be regular. The conditions are also sufficient. For, since $\left\{\beta_{p}(u)\right\}$ is totally monotone, the sequence $\left\{\Delta^{p} \beta_{0}(u)\right\}$ is monotone non-increasing, and therefore $\lim _{n=\infty} \Delta^{n} a_{0}=\lim _{n=\infty} \int_{0}^{1} \Delta^{n} \beta_{0}(u) d \alpha(u)=0$, so that $\left[H, a_{p}\right]$ is essentially regular when $(2.4)$ holds. Since $\beta_{0}(u) \not \equiv 0$ the manifold must contain at least one regular mean.

THEOREM 2.3. Let $\left\{\beta_{p}(u)\right\}, \beta_{0}(u) \not \equiv 0$, be a basis given by (2.1), where $B(u, t)$ is of bounded variation in $t, 0 \leqq t \leqq 1$, for each $u, 0 \leqq u \leqq 1$. Let $|B(u, t)|<K$, $0 \leqq t \leqq 1,0 \leqq u \leqq 1$, where $K$ is a finite constant independent of $u$ and $t$; and let $\lim _{t=0+} B(u, t)=B(u, 0)=0$ uniformly for $0 \leqq u \leqq 1$. Then $M\left[\beta_{p}(u)\right]$ is a regular manifold.

Proof. Let $a_{n}$ be given by (2.3). Then we shall prove that $\lim _{n=\infty} \Delta^{n} a_{0}=0$. We have

$$
\Delta^{n} a_{0}=\int_{0}^{1} \Delta^{n} \beta_{0}(u) d \alpha(u)=\int_{0}^{1} \int_{0}^{1}(1-t)^{n} d_{t} B(u, t) d \alpha(u) .
$$

Denote the inner integral by $I$. Then $I=I_{1}+I_{2}$ where $I_{1}=\int_{0}^{s}(1-t)^{n} d_{t} B(u, t)$, $I_{2}=\int_{s}^{1}(1-t)^{n} d_{t} B(u, t)$. After an integration by parts in $I_{1}$ we get

Consequently,

$$
I_{1}=(1-s)^{n} B(u, s)-\int_{0}^{8} B(u, t) d_{t}\left[(1-t)^{n}\right] .
$$

$$
\begin{aligned}
\left|I_{1}\right| & \leqq(1-s)^{n}|B(u, s)|+\underset{0 \leqq t \leqq s}{\text { l.u.b. }}|B(u, t)| \int_{0}^{8}\left|d_{t}(1-t)^{n}\right| \\
& \leqq(1-s)^{n} \epsilon+\left[1-(1-s)^{n}\right]_{\epsilon}=\epsilon,
\end{aligned}
$$

for all $u, 0 \leqq u \leqq 1$, provided only that $s<s_{v}$ where $s_{0}$ is sufficiently small.

Having chosen $s<s_{0}$, we integrate by parts in $I_{2}$ and get

$$
\begin{aligned}
I_{2} & =-(1-s)^{n} B(u, s)-\int_{s}^{1} B(u, t) d_{t}(1-t)^{n}, \\
\left|I_{2}\right| & \leqq(1-s)^{n}|B(u, s)|+\operatorname{liu.b.}_{s \leqq t \leqq 1}|B(u, t)| \int_{s}^{1}\left|d_{t}(1-t)^{n}\right| \\
& \leqq(1-s)^{n} \epsilon+K(1-s)^{n}=(1-s)^{n}(K+\epsilon) .
\end{aligned}
$$

Hence, if $n_{0}$ is sufficiently large, $\left|I_{2}\right|<\epsilon$ if $n>n_{0}$.

(7) This theorem holds for any basis such that $\left\{\Delta^{m} \beta_{0}(u)\right\}$ is uniformly bounded on $0 \leqq u \leqq 1$. 
We then have

$$
\left|\Delta^{n} a_{0}\right| \leqq \int_{0}^{1}\left(\left|I_{1}\right|+\left|I_{2}\right|\right)|d \alpha(u)| \leqq 2 \epsilon T, \quad n>n_{0},
$$

where $T$ is the total variation of $\alpha(u)$ in $0 \leqq u \leqq 1$. Thus $\lim _{n=\infty} \Delta^{n} a_{0}=0$, as was to be proved. Hence, every mean in $M\left[\beta_{p}(u)\right]$ is essentially regular; and since $\beta_{0}(u) \not \equiv 0$ it must contain at least one regular mean.

We now have the following comparison theorem.

THEOREM 2.4. Let $\left\{\beta_{p}(u)\right\}$ be a sequence of continuous real functions on the interval $0 \leqq u \leqq 1, \beta_{0}(u) \not \equiv 0$. Then $\left\{\beta_{p}(u)\right\}$ is a basis if there exists a totally monotone basis $\left\{\pi_{p}(u)\right\}$ such that $\Delta^{m} \beta_{n}(u) \leqq \Delta^{m} \pi_{n}(u), 0 \leqq u \leqq 1, m, n=0,1,2, \cdots$. Moreover, if $M\left[\pi_{p}(u)\right]$ is regular, and $\lim _{n=\infty} \Delta^{n} \beta_{0}(u)=0,0 \leqq u \leqq 1$, then $M\left[\beta_{p}(u)\right]$ is regular.

Proof. Put $\alpha_{p}(u)=\pi_{p}(u)-\beta_{p}(u)$. Then $\Delta^{n} \alpha_{p}(u)=\Delta^{n} \pi_{p}(u)-\Delta^{n} \beta_{p}(u) \geqq 0$, so that $\left\{\alpha_{p}(u)\right\}$ is a totally monotone basis. Thus, $\beta_{p}(u)=\pi_{p}(u)-\alpha_{p}(u)$ where $\left\{\pi_{p}(u)\right\}$ and $\left\{\alpha_{p}(u)\right\}$ are totally monotone bases. It readily follows that $\left\{\beta_{p}(u)\right\}$ is a basis. If $M\left[\pi_{p}(u)\right]$ is regular, so that $\lim _{n=\infty} \Delta^{n} \pi_{0}(u)=0$, and if $\lim _{n=\infty} \Delta^{n} \beta_{0}(u)=0$, then it follows that $\lim _{n=\infty} \Delta^{n} \alpha_{0}(u)=0$. Hence if $a_{n}=\int_{0}^{1} \beta_{n}(u) d \phi(u), \phi(u) \in B V[0,1]$, we must have $\lim _{n=\infty} \Delta^{n} a_{0}=0$, so that every mean in $M\left[\beta_{p}(u)\right]$ is essentially regular. The condition $\beta_{0}(u) \not \equiv 0$ insures that $M\left[\beta_{p}(u)\right]$ contains at least one regular mean. This completes the proof of the theorem.

Definition. $A$ manifold $M\left[\beta_{p}(u)\right]$ is said to include a given regular mean $\left[H, b_{p}\right]$ if any series which $\left[H, b_{p}\right]$ sums is also summed by every regular mean in $M\left[\beta_{p}(u)\right]$.

TheOREM 2.5. Let $M\left[\beta_{p}(u)\right]$ be regular, and $\left[H, b_{p}\right]$ a regular mean for which $b_{p} \neq 0, p=0,1,2, \cdots$. Then $M\left[\beta_{p}(u)\right]$ includes $\left[H, b_{p}\right]$ if and only if $\left\{\beta_{p}(u) / b_{p}\right\}$ is the basis for a regular manifold.

This follows at once from the theorem of Hausdorff [7] that a regular mean $\left[H, a_{p}\right]$ includes a regular mean $\left[H, b_{p}\right]$ for which $b_{p} \neq 0, p=0,1,2, \cdots$, if and only if $\left\{a_{p} / b_{p}\right\}$ is a moment sequence and $\left[H, a_{p} / b_{p}\right]$ a regular mean.

ILLUSTRATIVE EXAMPLES. Let $\beta_{n}^{(k)}(u)=(u+1) /\left[u+(n+1)^{k}\right], n=0,1$, $2, \cdots$, where $k$ is a positive integer. The sequence is totally monotone when $k=1$, and $\beta_{n}^{(1)}(u)=\int_{0}^{1} t^{n} d_{t} B_{1}(u, t), B_{1}(u, t)=t^{u+1}$. By Theorem 2.2, this is the basis of a regular manifold $M_{1}$.

When $k=2$, we find $B_{2}(u, t)=t\left[\cos \left(u^{1 / 2} \log t\right)-u^{-1 / 2} \sin \left(u^{1 / 2} \log t\right)\right]$, so that the sequence is not totally monotone. Since $d_{t} B_{2}(u, t)=-u^{-1 / 2}(1+u)$ $\cdot \sin \left(u^{1 / 2} \log t\right) d t$, it follows that $\int_{0}^{t}\left|\cdot d_{t} B_{2}(u, t)\right| \leqq 2,0 \leqq u \leqq 1,0 \leqq t \leqq 1$. Also, $\left|B_{2}(u, t)\right| \leqq t(1-\log t), 0 \leqq t \leqq 1$. It therefore follows from Theorems $2.1,2.3$ that $M\left[\beta_{p}^{(2)}(u)\right]$ is a regular manifold. 
On writing $\beta_{p}^{(k)}(u)$ as a sum of partial fractions it is now easy to see that $\left\{\beta_{p}^{(k)}(u)\right\}$ is a basis for a regular manifold $M_{k}, k=1,2,3, \cdots$.

To illustrate Theorem 2.5 , we shall show that $M_{1}$ includes $(C, 1)$ but does not include $(C, k)$ if $k>1$. The sequence $\left\{\beta_{n}^{(1)}(u) / b_{n}\right\}, b_{n}=1 /(1+n)$, must be proved to be a basis for a regular manifold. We find that

$$
\beta_{n}^{(1)}(u) / b_{n}=(u+1)(n+1) /(u+n+1)=\int_{0}^{1} t^{n} d_{t} Q(u, t),
$$

where $Q(u, t)=-u t^{u+1}, 0 \leqq t<1$, and $Q(u, 1)=1$. By Theorem 2.3, this is the basis of a regular manifold, so that $M_{1}$ includes $(C, 1)$. On the other hand, if $b_{n}=(1+n)^{-k}, k>1$, then $\beta_{n}^{(1)}(u) / b_{n}$ tends to $\infty$ with $n$, so that $M_{1}$ cannot include $(C, k), k>1$.

We shall give an elementary proof that $M_{k}$ includes $(C, k)$ but not $(C, k+\theta), \theta>0$. An arbitrary mean in $M_{k}$ has the form $\left[H, b_{n}\right]$ where

$$
\begin{aligned}
b_{n} & =\left[\frac{c_{0}}{(n+1)^{k}}+\frac{(n+1)^{k}-1}{(n+1)^{k}}\left(\frac{c_{1}}{(n+1)^{k}}-\frac{c_{2}}{(n+1)^{2 k}}+\cdots\right)\right], \\
c_{n}=\int_{0}^{1} u^{n} d \phi(u), & \phi(u) \in B V[0,1] .
\end{aligned}
$$

We observe that the mean $\left[H, b_{n}^{*}\right]$ obtained by replacing $c_{n}$ by $c_{n+1}$, $n=0,1,2, \cdots$, is also in $M_{k}$. This amounts to using $u d \phi(u)$ instead of $d \phi(u)$. Of course $\left[H, b_{n}\right]$ is regular if and only if $b_{0}=1$, i.e., $c_{0}=1$.

To prove that $\left[H, b_{n}\right] \supset(C, k)$ we must show that the sequence $\left\{(n+1)^{k} b_{n}\right\}$ is a regular sequence $\left({ }^{8}\right)$ when $c_{0}=1$. We have

$$
(n+1)^{k} b_{n}=c_{0}+c_{1}-\left[\frac{c_{1}}{(n+1)^{k}}+\frac{(n+1)^{k}-1}{(n+1)^{k}}\left(\frac{c_{2}}{(n+1)^{k}}-\frac{c_{3}}{(n+1)^{2 k}}+\cdots\right)\right] .
$$

On comparing this with (2.5) we see that $(n+1)^{k} b_{n}=r \cdot 1+s \cdot b_{n}^{*}, r+s=1$, is a linear combination of regular sequences, where the constants of combination add up to unity, and is therefore a regular sequence. Hence we have proved that $\left[H, b_{n}\right] \supset(C, k)$. Since, in general, $(n+1)^{k+\theta} b_{n} \rightarrow \infty$ as $n \rightarrow \infty$ if $\theta>0$, it follows that $\left[H, b_{n}\right]$ does not include $(C, k+\theta)$.

3. The manifold $M\left[(1+p u)^{-1}\right]$. The sequence $\mu_{p}=(1+p u)^{-1}, p=0,1,2, \cdots$, is a totally monotone basis inasmuch as $\mu_{p}(u)$ is continuous and $\Delta^{m} \mu_{n} \geqq 0$, $m, n=0,1,2, \cdots, 0 \leqq u \leqq 1$. Since $\lim _{n=\infty} \Delta^{n} \mu_{0}=0,0 \leqq u \leqq 1$, the manifold $M\left[\mu_{p}(u)\right]$ is regular, by Theorem 2.2. This can be established also by Theorem 2.3. For, if $M(u, t)$ is the function defined as follows:

$$
M(u, t)=\left\{\begin{array}{lrr}
t^{1 / u}, & 0<u \leqq 1, & 0 \leqq t \leqq 1, \\
0, & u=0, & 0 \leqq t<1, \\
1, & u=0, & t=1,
\end{array}\right.
$$

$\left.{ }^{8}\right)$ A regular sequence is one which determines a regular mean. 
then $\mu_{p}(u)=\int_{0}^{1} t^{p} d_{t} M(u, t), p=0,1,2, \cdots$. It is readily seen that (3.1) satisfies the conditions imposed upon $B(u, t)$ in Theorem 2.3.

We shall begin by determining a large class of means which are in this manifold. Let

$$
a_{n}=\int_{0}^{1} \frac{d \alpha(u)}{1+n u}, \quad n=0,1,2, \cdots, \alpha(u) \in B V[0,1],
$$

so that $\left[H, a_{n}\right]$ is a mean in $M\left[\mu_{p}(u)\right]$. Since $\left\{a_{n}\right\}$ is a moment sequence, there must exist a function $\theta(t)$ in $B V[0,1]$ such that

$$
a_{n}=\int_{0}^{1} t^{n} d \theta(t), \quad n=0,1,2, \cdots .
$$

One may determine $\theta(t)$ in the following way. Put $p(x)=a_{0}-a_{1} x+a_{2} x^{2}-\cdots$. Then, on putting in the values of the $a_{n}$ 's from (3.2) and using a well known integral representation for hypergeometric functions, we get

$$
p(x)=\int_{0}^{1} \frac{d \theta(t)}{1+x t}=\int_{0}^{1} \int_{0}^{1} \frac{d_{t} M(u, t)}{1+x t} d \alpha(u)=\int_{0}^{1} \frac{d_{t} \int_{0}^{1} M(u, t) d \alpha(u)}{1+x t},
$$

where $M(u, t)$ is given by (3.1), and where the last step may be verified directly from the definition of a Stielties integral. Consequently,

$$
\theta(t)=\int_{0}^{1} M(u, t) d \alpha(u), \quad 0 \leqq t \leqq 1,
$$

We shall now prove the following theorem.

THEOREM 3.1. Let $\theta(t)=p_{1} t+p_{2} t^{2}+p_{3} t^{3}+\cdots$ be any power series with constant term 0 and which is absolutely convergent when $t=1$. Then $\theta(t) \in B V[0,1]$, and $[H, \theta(t)]$ is a mean in $M\left[(1+p u)^{-1}\right]$.

Proof. Let

$$
\alpha(u)=\left\{\begin{array}{rcc}
p_{1}+p_{2}+p_{3}+p_{4}+\cdots, & 1 \leqq u, \\
p_{2}+p_{3}+p_{4}+\cdots, & 1 / 2 \leqq u<1, \\
p_{3}+p_{4}+\cdots, & 1 / 3 \leqq u<1 / 2, \\
\cdots, & \cdots, \\
0, & u=0 .
\end{array}\right.
$$

Then $\alpha(u) \in B V[0,1]$; and if this function is used in (3.2) there is determined a mean $\left[H, a_{n}\right]$ in $M\left[\mu_{p}(u)\right]$. We see by (3.3), (3.4) that $\left[H, a_{n}\right]=[H, \theta(t)]$ where $\theta(t)=p_{1} t+p_{2} t^{2}+\cdots$. This proves the theorem.

The function $1-(1-t)^{\alpha}, \Re(\alpha)>0$, which is the mass function for Cesàro summability $(C, \alpha)$, has a power series of the kind specified in Theorem 3.1, and hence $(C, \alpha)$ is in the manifold $M\left[\mu_{p}(u)\right]$. 
Throughout the remainder of this section we shall consider only the subset of means of $M\left[\mu_{p}(u)\right]$ of the form $[H, g(p)]$ where

$$
g(x)=\int_{0}^{1} \frac{d \phi(u)}{1+x u}, \quad \phi(u) \text { monotone, } \phi(1)-\phi(0)=1 .
$$

We recall that $g(x)$ has a continued fraction representation of the form [11]

$$
g(x)=\frac{1}{1}+\frac{g_{1} x}{1}+\frac{\left(1-g_{1}\right) g_{2} x}{1}+\frac{\left(1-g_{2}\right) g_{3} x}{1}+\cdots,
$$

where $0 \leqq g_{n} \leqq 1, n=1,2,3, \cdots$, it being agreed that if some $g_{n}$ is 0 or 1 the continued fraction terminates with the first identically vanishing partial quotient. Conversely, any such continued fraction represents a function of the form (3.5). The function $g(x)$ is holomorphic in the plane of $x$ cut along the real axis from $x=-1$ to $x=-\infty$.

Theorem 3.2. $(C, 0) \subset[H, g(p)] \subset(C, 1)$.

Proof. Since $[H, g(p)]$ is regular, it includes $(C, 0)$ (convergence). To prove the second relation, put $g^{*}(x)=1 / 1+\left(1-g_{1}\right) x / 1+g_{1}\left(1-g_{2}\right) x / 1+\cdots$, the continued fraction being obtained from (3.6) by replacing $g_{n}$ by $1-g_{n}$, $n=1,2,3, \cdots$. Then, we have the continued fraction identity $[11, \mathrm{p} .166]$

$$
g(x) g^{*}(x)=1 /(1+x), \quad \text { all } x \text { not real and } \leqq-1 .
$$

Since $\left[H, g^{*}(p)\right]$ is a regular mean, it follows that $[1 /(1+p)]: g(p), p=0,1$, $2, \cdots$, is a regular sequence, so that, since $(C, 1)=[H, 1 /(1+p)]$, the inclusion relation follows by Hausdorff's fundamental theorem.

It is perhaps of interest to determine conditions under which $[H, g(p)]$ $\approx(C, 0),[H, g(p)] \approx(C, 1)$. We have this theorem.

THEOREM 3.3. If $g(x)$ is the function (3.5), then $[H, g(p)] \approx(C, 0)$ if and only if $\phi(u)$ is discontinuous at $u=0$; and $[H, g(p)] \approx(C, 1)$ if and only if $\int_{0}^{1} d \phi(u) / u$ is finite.

Proof. From the equation

$$
(n+1) \int_{0}^{1} \frac{d \phi(u)}{1+n u}=\left(1+\frac{1}{n}\right) \int_{0}^{1} \frac{d \phi(u)}{(1 / n)+u}, \quad n>0,
$$

it follows that $\{(n+1) g(n)\}$ is a bounded sequence if and only if $\int_{0}^{1} d \phi(u) / u$ converges. Moreover, when $\{(n+1) g(n)\}$ is bounded, it is a regular moment sequence. For, in terms of the bounded monotone function $\psi(u)=\int_{0}^{u}(1-t) d \phi(t) / t$, we have

$$
(n+1) g(n)=\int_{0}^{1} \frac{d \phi(u)}{u}-\int_{0}^{1} \frac{d \psi(u)}{1+n u},
$$


and thus have expressed our sequence as a linear combination of two regular sequences, where the constants of combination add up to unity. Hence we have shown that $g(p):(1+p)^{-1}, p=0,1,2, \cdots$, is a regular sequence, i.e., $[H, g(p)] \supset(C, 1)$, if and only if $\int_{0}^{1} d \phi(u) / u<\infty$. Since we previously had $[H, g(p)] \subset(C, 1)$, the second part of the theorem is proved

To prove the first part, we use (3.7) and write $1 / g(p)=(p+1) g^{*}(p)$. Hence, from the foregoing proof, $\{1 / g(p)\}$ is a regular sequence if (and only if) it is bounded, i.e., if and only if

$$
\lim _{n=\infty} g(n)=\lim _{n=\infty} \int_{0}^{1} \frac{d \phi(u)}{1+n u}>0 .
$$

But this well known limit is equal to $\phi(0+)-\phi(0)$, the discontinuity of $\phi(u)$ at $u=0$. This completes the proof of the theorem.

From the above proof we see that an alternative form of Theorem 3.3 is as follows.

THEOREM 3.4. If $g(x)$ is the function (3.5), then $[H, g(p)] \approx(C, 0)$ if and only if the sequence $\{1 / g(p)\}$ is bounded; and $[H, g(p)] \approx(C, 1)$ if and only if the sequence $\{(p+1) g(p)\}$ is bounded.

The conditions may be formulated in terms of the continued fraction (3.6) for $g(x)$ as follows.

THEOREM 3.5. If $g(x)$ is the function (3.5) with continued fraction representation (3.6), then $[H, g(p)] \approx(C, 0)$ if and only if the series obtained from

$$
1+\sum_{p=1}^{\infty} \frac{g_{1} g_{2} \cdots g_{p}}{\left(1-g_{1}\right)\left(1-g_{2}\right) \cdots\left(1-g_{p}\right)}
$$

by replacing $g_{2 k}$ by $1-g_{2 k}, k=1,2,3, \cdots$, converges; and $[H, g(p)] \approx(C, 1)$ if and only if the series obtained from (3.8) by replacing $g_{2 k-1}$ by $1-g_{2 k-1}$, $k=1,2,3, \cdots$, converges.

Proof. Let $G(x), H(x)$ be the functions obtained from (3.6) by substituting $1-g_{2 k}$ for $g_{2 k}$ and $1-g_{2 k-1}$ for $g_{2 k-1}$, respectively, $k=1,2,3, \cdots$. Then [12] $G\left(-x[1+x]^{-1}\right)=1 / g(x), H\left(-x[1+x]^{-1}\right)=(1+x) g(x)$. As $x \rightarrow+\infty$, we see that $-x /(1+x) \rightarrow-1^{+}$. The conditions stated are necessary and sufficient $[11$, p. 181] for $G(x)$ and $H(x)$, respectively, to remain finite as $x \rightarrow-1$ through real values greater than -1 . Hence the theorem follows by Theorem 3.4.

EXAMPLES. If $g_{p}=1 / 2, p=1,2,3, \cdots$, in (3.6), then $g(p)=(1+p)^{-1 / 2}$, and therefore $[H, g(p)]=[H, 1 / 2] \approx(C, 1 / 2)$. If $g_{2 k-1}=1 / 2, g_{2 k}=2 / 3$ then $[H, g(p)] \approx(C, 0)$; while if $g_{2 k-1}=2 / 3, g_{2 k}=1 / 2$ then

$$
[H, g(p)] \approx(C, 1) .
$$

If the coefficients $p_{1}, p_{2}, p_{3}, \cdots$ in the power series of Theorem 3.1 are 
real and greater than or equal to 0 , and $\sum p_{n}=1$, then $[H, \theta(t)]$ is a mean of the kind being considered. In particular, if $\theta(t)=1-(1-t)^{\alpha}, 0 \leqq \alpha \leqq 1$, the coefficients are greater than or equal to 0 and their sum is unity, and consequently the Cesàro means $(C, \alpha), 0 \leqq \alpha \leqq 1$, are all of the form $[H, g(p)]$ where $g(x)$ is of the form (3.5).

4. Inclusion problems in the difference matrix for $\{g(p)\}$. If $\left\{c_{n}\right\}$ is a moment sequence, then the row, column, and diagonal sequences in the difference matrix [5]

$$
\left(\Delta^{m} c_{n}\right)=\left(\begin{array}{cccc}
c_{0}, & c_{1}, & c_{2}, & \ldots \\
\Delta c_{0}, & \Delta c_{1}, & \Delta c_{2}, & \ldots \\
\Delta^{2} c_{0}, & \Delta^{2} c_{1}, & \Delta^{2} c_{2}, & \ldots \\
\cdot & . & . & \ldots
\end{array}\right)
$$

are all moment sequences, and accordingly define Hausdorff means. The following theorem concerns the rows in this matrix when the base sequence $\left\{c_{p}\right\}$ is of the form $\{g(p)\}$. Its proof affords an application of some of the ideas of $\S 2$.

THEOREM 4.1. Let $g(x)$ be a function of the form

$$
g(x)=\int_{0}^{1} \frac{d \phi(u)}{1+x u}, \quad \phi(u) \in B V[0,1] .
$$

Put $b_{n}=\Delta^{m} g(n) / \Delta^{m} g(0)$, supposing $\Delta^{k} g(0) \neq 0, k=0,1,2, \cdots$ Then $\left[H, b_{n}\right]$ is a regular mean, and

$$
\left[H, b_{n}\right] \supset(C, m), \quad m=1,2,3, \cdots .
$$

Proof. Since $\lim _{n=\infty} \Delta^{n} g(0)=0$ it follows that $\lim _{n=\infty} \Delta^{n} b_{0}=0$. Thus $\left[H, b_{n}\right]$ is regular. We use Theorems $2.5,2.3$ to prove (4.2). To do this, we note that $\left\{\beta_{p}(u)\right\}$, where

$$
\beta_{p}(u)=\Delta^{m}(1+p u)^{-1}=m ! u^{m} /(1+p u)(1+[p+1] u) \cdots(1+[p+m] u),
$$

is a totally monotone basis for a regular manifold, $M\left[\beta_{p}(u)\right]$, and that the mean $\left[H, b_{n}\right]$ is in $M\left[\beta_{p}(u)\right]$. To prove (4.2) it is sufficient, by Theorem 2.5, to prove that the sequence $\left\{\beta_{p}(u) / c_{p}\right\}$, where $c_{p}=G_{p+m, m}, p=0,1,2, \cdots$, is the moment sequence defining $(C, m)$, is the basis for a regular manifold; and this will in turn be true if

$$
\gamma_{n}(u)=C_{n+m} \beta_{n}(u)=\int_{0}^{1} t^{n} d \imath B(u, t), \quad n=0,1,2, \cdots,
$$

where $B(u, t)$ satisfies the conditions of Theorem 2.3.

Since $\gamma_{n}(0)=0$, we must have $B(0, t) \equiv 0,0 \leqq t \leqq 1$.

If $0<u \leqq 1$, we find that 


$$
\gamma_{n}(u)=\frac{A_{0}}{1+n u}+\frac{A_{1}}{1+(n+1) u}+\cdots+\frac{A_{m}}{1+(n+m) u},
$$

where $u^{m} A_{k}$ is a polynomial in $u$ of degree $m$ with coefficients independent of $n$. Hence it follows that if $u>0, \gamma_{n}(u)$ is a linear combination of totally monotone sequences with coefficients of combination independent of $n$. Therefore $\left\{\gamma_{n}(u)\right\}$ is a moment sequence. Hence there exists a function $B(u, t)$ in $B V[0,1]$ such that $(4.3)$ holds, $0 \leqq u \leqq 1$.

We show next that

$$
\lim _{t=0^{+}} B(u, t)=0, \quad \text { uniformly for } 0 \leqq u \leqq 1 .
$$

If $u>0$, it follows from (4.4) that $B(u, t)$ can be expressed as the sum of $m+1$ functions of the form $\left[h(u) t^{p} t^{1 / u}\right] / u^{m}, p$ an integer, $0 \leqq p \leqq m$, where $h(u)$ is a polynomial in $u$. Hence it is easy to see that $|B(u, t)|<k t$, where $k$ is a constant independent of $u, t$, provided that $0<t \leqq r<1, r$ a fixed number less than $1,0<u \leqq 1$. It follows that (4.5) holds.

It remains to be shown that

$$
|B(u, t)| \leqq K, 0 \leqq u \leqq 1,0 \leqq t \leqq 1, K<\infty \text { and independent of } u, t .
$$

The proof is by induction starting with $m=0$, for which the requirement is clearly met. If $u>0,\left\{\gamma_{n}(u) f(m, u)\right\}$ is a regular sequence if $f(m, u)$ is chosen so that $\gamma_{0} f(m, u)=1$. We shall use the superscript $m$ to indicate dependence upon $m$.

To prove (4.6) by induction, we assume that

$$
\left|B^{(m)}(u, t)\right| \leqq K^{(m)}, \quad 0 \leqq u \leqq 1,0 \leqq t \leqq 1, m \geqq 0,
$$

and shall prove it for $m+1$. We have

$$
\gamma_{n}^{(m+1)}(u) f(m+1, u)=\frac{(n+m+1) u}{1+(n+m+1) u} e(m, u) \cdot \gamma_{n}^{(m)}(u) f(m, u),
$$

where $e(m, u)=[1+(m+1) u] /(m+1) u$. Then, we may write

$$
\begin{aligned}
\frac{(n+m+1) u}{1+(n+m+1) u} e(m, u) & =\int_{0}^{1} t^{n} d_{t} E(u, t), & & n=0,1,2, \cdots, \\
\gamma_{n}^{(m)}(u) f(m, u) & =\int_{0}^{1} t^{n} d_{t} F(u, t), & & n=0,1,2, \cdots,
\end{aligned}
$$

which are both regular sequences if $u>0$.

We now use a composition theorem $[4$, p. 201] for these integrals and get

$$
\gamma_{n}^{(m+1)}(u) f(m+1, u)=\int_{0}^{1} t^{n} d_{t} E(u, t) \cdot \int_{0}^{1} t^{n} d_{t} F(u, t)=\int_{0}^{1} t^{n} d_{t} G(u, t),
$$


where

$$
G(u, t)=E(u, t)+\int_{\imath}^{1} F(u, t / v) d_{v} E(u, v)
$$

or

$$
f(m+1, u) B^{(m+1)}(u, t)=E(u, t)+\int_{t}^{1} f(m, u) B^{(m)}(u, t / v) d_{v} E(u, v) .
$$

Thus

$$
B^{(m+1)}(u, t)=h(u, t)+\int_{t}^{1} B^{(m)}(t, t / v) d_{v} E^{*}(u, v)
$$

where $E^{*}(u, v)=\left[v^{m+1+(1 / u)}\right] /[(m+1) u+1]$, and $h(u, t)$ is a bounded function $|h(u, t)| \leqq H, 0 \leqq u \leqq 1,0 \leqq t \leqq 1$. We therefore have

$$
\left|B^{(m+1)}(u, t)\right| \leqq H+K^{(m)} \int_{0}^{1} d_{v} E^{*}(u, v) \leqq K^{(m+1)}, \quad u>0,0 \leqq t \leqq 1,
$$

where $K^{(m+1)}$ is a finite constant. Since the function is identically 0 for $u=0$, the result holds also for $u=0$. This completes the induction and the proof of Theorem 4.1.

\section{Gronwall summability}

5. Introduction to Part II. T. H. Gronwall [6] introduced a very general method of summation, based upon two analytic functions, a miapping function $z=f(w)$, and a weight function $g(w)=\sum_{p=0}^{\infty} b_{p} w^{p}, b_{p} \neq 0$. The $n$th $(f, g)$-sum of the series $\sum u_{p}$ is $U_{n}$, where $U_{n}$ is determined from the power series identity in $w$

$$
\sum_{n=0}^{\infty} u_{n}[f(w)]^{n}=[g(w)]^{-1} \sum_{n=0}^{\infty} b_{n} U_{n} w^{n}
$$

The function $z=f(w)$ is regular for $|w| \leqq 1$ except possibly at $w=1$, and maps $|w|<1$ one-to-one upon a region $D$ interior to $|z|<1$. Under this mapping, $w=0$ corresponds to $z=0$ and $w=1$ to $z=1$. The inverse function $f^{-1}(z)$ is regular on the boundary of $D$ except possibly at $z=1$, and at this point

$$
1-w=(1-z)^{\lambda}\left[a+a_{1}(1-z)+\cdots\right], \quad \lambda \geqq 1, a>0 .
$$

The function $g(w)$ has the form

$$
g(w)=(1-w)^{-\alpha}+\gamma(w), \quad \alpha>0,
$$

where $\gamma(w)$ is regular for $|w| \leqq 1$; and $g(w) \neq 0$ for $|w|<1$.

The series $\sum u_{n}$ is said to be $(f, g)$-summable to $s$ if $\lim _{n=\infty} U_{n}=s$.

Special $(f, g)$-methods are $(C, k)$ for $k$ real and greater than $-1 ;(E, \beta)$ (Euler-Knopp) for $0<\beta \leqq 1$; de la Vallée Poussin summability $(V)$; and 
a generalized $(V)$-summability, $(V k)$, introduced by Gronwall. Recently C. Birindelli [1] has shown that a method of summation of Obrechkoff is the $(f, g)$-method for which $f=1-(1-w)^{1 / 2}, g=(1-w)^{-1 / 2}$. We show in $\S 7$ that a method of summation introduced by W. A. Mersman [8] is a special $(f, g)$-method.

Some of the important properties of $(f, g)$-summability are the following:

(a) If $(f, g),\left(f_{1}, g_{1}\right)$ are two Gronwall means with map regions $D, D_{1}$ and with exponents $\lambda, \lambda_{1}$ (cf. (5.2)), then $(f, g) \supset\left(f_{1}, g_{1}\right)$ if $\lambda>\lambda_{1}$, and $D$ is interior to $D_{1}$.

(b) If $\lambda>1$, then $(f, g) \supset(C, k), k>-1$.

(c) The exact domain in which $(f, g)$ sums the geometrical series $\sum x^{n}$ to the sum $1 /(1-x)$ is the interior of the region bounded by the curve

$$
x=1 / f\left(e^{i \theta}\right), \quad-\pi \leqq \theta \leqq+\pi .
$$

(d) If $\sum u_{n}$ is $(f, g)$-summable to $s$, then $\phi(z)=\sum u_{n} z^{n}$ is holomorphic inside the map region $D$, and $\phi(z) \rightarrow s$ uniformly as $z \rightarrow 1$ over every path of $z$ interior to $D$ which reaches 1 inside the sector $z=1-r e^{i \theta},-\theta_{0}<\theta<+\theta_{0}$, $\theta_{0}<\pi / 2 \lambda, r \geqq 0, \lambda$ defined in (5.2).

The properties (a), (b), (d) were established by Gronwall. Property (c) was established for $(V k)$ by Gronwall, and extended to the general case by C. Birindelli [2].

The main problem which we consider here is as follows: to determine all means which are common to the class of Hausdorff means and the class of Gronwall means. We find these to be the means $\left[H, c_{n}\right]$ where

$$
c_{n}=\beta^{n} / C_{n+\alpha, n}, \quad 0<\beta \leqq 1, \alpha \geqq 0,
$$

the Gronwall mean identical with this Hausdorff mean being $(f, g)$ where

$$
f(w)=\frac{\beta w}{1-(1-\beta) w}, \quad g(w)=(1-w)^{-\alpha-1} .
$$

6. Hausdorff means which are also Gronwall means. If we suppose that the Hausdorff mean $\left[H, c_{n}\right]$ is the Gronwall mean $(f, g)$, then if we put $u_{n}=0$ for $n \neq k, u_{k}=1$, in (5.1) we find the relation

$$
[f(w)]^{k}=[g(w)]^{-1} \sum_{n=k}^{\infty} \alpha_{n, k} w^{n}, \quad \alpha_{n, k}=\sum_{p=k}^{n} C_{n, p} \Delta^{n-p} c_{p},
$$

which must hold identically in $w$ for $k=0,1,2, \cdots$. We shall suppose that $\left[H, c_{n}\right]$ is regular, so that $c_{0}=1$. Then, by (6.1) with $k=1$, we have

$$
f(w)=[g(w)]^{-1} \sum_{n=0}^{\infty} b_{n}\left(1-\Delta^{n} c_{0}\right) w^{n} .
$$

To determine $b_{n}$, put $f(w)=a_{1} w+a_{2} w^{2}+\cdots$ in (6.1). Then we find the rela- 
tions $b_{n} c_{n}=b_{0} a_{1}^{n}, n=0,1,2, \cdots$. If $a_{1}=0$, then $c_{1}=c_{2}=\cdots=0$ inasmuch as $b_{n}$ must be different from 0 , and hence by (6.2) we would have $f(w) \equiv 0$ which is impossible since $f(1)=1$. Consequently, $a_{1} \neq 0$, and therefore $c_{n} \neq 0$, $n=1,2,3, \cdots$; and $b_{n}=b_{0} a_{1}^{n} / c_{n}, n=0,1,2, \cdots$. Now it is clear that (5.1) is unaltered if $b_{n}$ is replaced by $k b_{n}, n=0,1,2, \cdots$, where $k$ is any constant not 0 . It follows that there is no restriction in assuming that $b_{0}=1$. Hence we have the following necessary conditions for the (regular) Hausdorff mean $\left[H, c_{n}\right]$ to be the same as the Gronwall mean $(f, g)$ :
$c_{n} \neq 0$,
$n=0,1,2, \cdots, \quad c_{0}=1$
$(6.3$, ii $)$
$(6.3, \mathrm{iii})$

$$
g(w)=\sum_{n=0}^{\infty}(\theta w)^{n} / c_{n}
$$$$
f(w)=1-[g(w)]^{-1} \sum_{n=0}^{\infty} \Delta^{n} c_{0}(\theta w)^{n} / c_{n},
$$

where $\theta$ is a parameter unequal to 0 so chosen that $f(1)=1$.

To obtain other necessary conditions, divide both members of (5.1) by $1-f(w)$, put $s_{n}=u_{0}+u_{1}+\cdots+u_{n}=0, n \neq k, s_{k}=1, U_{n}=\sum_{p=0}^{n} C_{n, p} \Delta^{n-p} c_{p} \cdot s_{p}$, $\theta w=t, f(w)=F(t)$, and we obtain the equation

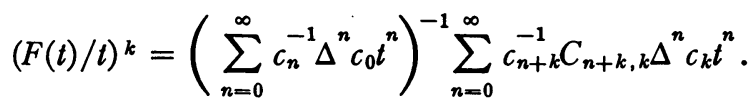

This obviously holds when $k=0$. If it is to hold for all $k$ it must hold for $k+1$ when it holds for $k$. Hence, on multiplying both members of $(6.4)$ by $F(t) / t$ we find that a necessary and sufficient condition for (6.4) to hold for all $k$ is that

$$
\begin{aligned}
\sum_{n=0}^{\infty} c_{n+1}^{-1}(1- & \left.\Delta^{n+1} c_{0}\right) t^{n} \cdot \sum_{n=0}^{\infty} c_{n+k}^{-1} C_{n+k, k} \Delta^{n} c_{k} t^{n} \\
& \equiv \sum_{n=0}^{\infty} c_{n}^{-1} t^{n} \cdot \sum_{n=0}^{\infty} c_{n+k+1}^{-1} C_{n+k+1, k+1} \Delta^{n} c_{k+1} t^{n}, \quad k=0,1,2, \cdots
\end{aligned}
$$

On equating the coefficients of the first power of $t$ on either side we obtain the relation

$$
c_{k+1}^{-1}(k+1)\left(c_{k}-c_{k+1}\right)+c_{2}^{-1}\left(2 c_{1}-c_{2}\right)=c_{k+2}^{-1}(k+2)\left(c_{k+1}-c_{k+2}\right)+c_{1}^{-1} .
$$

This serves as a recursion relation to determine $c_{k}$ parametrically, whence we find that $c_{k}$ must have the form $\beta^{k} / C_{k+\alpha, k}$.

When this value of $c_{k}$ is substituted in (ii), (iii) of (6.3) we get: $F(t)=\beta t /[\beta-(1-\beta) t]=f(t / \theta), f(w)=\beta w \theta /[\beta-(1-\beta) w \theta]$. If we take $\theta=\beta$ we find for $f(w), g(w)$ the values (5.4). If these functions are to satisfy the conditions of Gronwall we must have, first of all, $\alpha$ real and $\alpha+1>0$. But since 
$\left[H, c_{n}\right]$ is a regular Hausdorff mean, $\Re(\alpha) \geqq 0$. Hence we must have $\alpha$ real and greater than or equal to 0 .

We now determine $\beta$ so that the map of $|w|<1$ by $z=f(w)$ shall lie in $|z|<1$. Put $w=e^{i \theta}, \beta=p+i q$, and we see that $|z| \leqq 1$ if and only if $p+[q / \tan (\theta / 2)] \leqq 1$; and hence $q=0, \beta=p \leqq 1$. The map of $|w|=1$ is a circle in the $z$-plane whose intercepts on the real axis are $z=-\beta /(2-\beta), z=1$. In order that this map should contain the origin it is necessary that $\beta \geqq 0$. Since $\beta=0$ is clearly excluded, we therefore have $0<\beta \leqq 1$.

We have shown that $c_{n}$ must have the form (5.3). Consequently

$$
c_{n}=\int_{0}^{1} u^{n} d \phi(u), \quad n=0,1,2, \cdots,
$$

where

$$
\phi(u)= \begin{cases}1-\left(1-u \beta^{-1}\right)^{\alpha}, & u \leqq \beta, \\ 1, & u>\beta .\end{cases}
$$

Thus $\left[H, c_{n}\right]$ is a regular Hausdorff mean.

It remains to be proved that when $c_{n}$ is given by (6.6) then (6.4) holds for all values of $k$. On making use of the integral representation (6.6) we find that (6.4) will hold if

$$
\left[\frac{1-w}{1-(1-\beta) w}\right]^{k+1}=\alpha C_{k+\alpha, k} \int_{0}^{1} \frac{u^{k}(1-u)^{\alpha-1} d u}{\left[1+\beta w(1-w)^{-1} u\right]^{k+\alpha+1}} .
$$

But the right member is equal to

$$
\alpha C_{k+\alpha, k} \frac{\Gamma(k+1) \Gamma(\alpha)}{\Gamma(k+\alpha+1)} \sum_{n=0}^{\infty} C_{n+k, n}[-\beta w /(1-w)]^{n}=\left[1+\beta w(1-w)^{-1}\right]^{-k-1},
$$

which is equal to the left member. It now follows that $\left[H, c_{n}\right]$ is the Gronwall mean $(f, g)$, where $f, g$ are given by (5.4).

We have proved the following theorem.

THEOREM 6.1. A necessary and sufficient condition in order for a regular Hausdorff mean $\left[H, c_{n}\right]$ to be a Gronwall mean $(f, g)$ is that

$$
c_{n}=\beta^{n} / C_{n+\alpha, n}=\int_{0}^{1} u^{n} d \phi(u), \quad n=0,1,2, \cdots, \alpha \geqq 0,0<\beta \leqq 1,
$$

where $\phi(u)=1-\left(1-u \beta^{-1}\right)^{\alpha}$ if $u \leqq \beta, \phi(u)=1$ if $u>\beta$. The Gronwall mean which is the same as this has $f, g$ given by (5.4).

We note that the domain in which this mean sums the geometrical series, and which is given by (c) of $\S 5$, is the circular region $|z+(1-\beta) / \beta|<1 / \beta$. This result furnishes additional evidence in support of the conjecture $[5, \mathrm{p}$. 
205] that a necessary and sufficient condition that a Hausdorff mean sum a power series outside its circle of convergence is that the mass function be constant in the neighborhood of 1 .

7. Mersman summability. In a recent paper, W. A. Mersman [8] studied the transformation

$$
U_{n}=\left(\frac{1}{2}\right)^{2 n} \sum_{p=0}^{n} C_{2 n+k, n-p} \cdot\left(u_{0}+u_{1}+\cdots+u_{p}\right), \quad n=0,1,2, \cdots .
$$

He proved that this defines a regular method of summation, which we shall call $(M)$-summability. He found that $(M) \supset(C, k)$ for $k=1,2 ;(M)$ includes an Euler-Knopp method; and he determined the domain in which $(M)$ sums the geometrical series. We shall now prove the following theorem.

TheOREM 7.1. (M)-summability is $(f, g)$-summability with

$$
f(w)=\frac{1-(1-w)^{1 / 2}}{1+(1-w)^{1 / 2}}, \quad g(w)=(1-w)^{-1} .
$$

Proof. It is required to show that when these values of $f(w), g(w)$, and $U_{n}$ from (7.1) are substituted in (5.1), the latter becomes a power series identity. This can be done in exactly the same way that Gronwall established the corresponding theorem for de la Vallée Poussin summability $(V)$ determined by

$$
U_{n}=\sum_{p=0}^{n} \frac{(n !)^{2}}{(n-p) !(n+p) !} u_{p}, \quad n=0,1,2, \cdots,
$$

with $f(w), g(w)$ given by

$$
f(w)=\frac{1-(1-w)^{1 / 2}}{1+(1-w)^{1 / 2}}, \quad g(w)=(1-w)^{-1 / 2} .
$$

On making the indicated substitutions, and putting $u_{n}=0, n \neq k, u_{k}=1$, we find that the following identity must be verified

$$
(1-w)^{-1}[f(w)]^{k}=\sum_{n=k}^{\infty}\left(\frac{1}{2}\right)^{2 n} \sum_{p=k}^{n} C_{2 n+1, n-p} w^{n}, \quad k=0,1,2, \cdots .
$$

One may show directly that this holds for $k=0,1$. Then by means of the identity

$$
[f(w)]^{k+1}=[f(w)]^{k}\left(4 w^{-1}-2\right)-[f(w)]^{k-1}, \quad k=1,2,3, \cdots,
$$

one may prove (7.5) by mathematical induction for all values of $k$. On substituting (7.5) in (5.1) the proof may be completed exactly as in Gronwall's proof.

On comparing (7.2) and (7.4) we see that the map function $f(w)$ is the same for $(M)$ - and $(V)$-summabilities, while $g(w)$ is of the form $(1-w)^{-\alpha}$ for 
both methods, with $\alpha$ having a larger value in the case of $(M)$-summability. It therefore follows from a theorem of Birindelli [2] that $(V) \subset(M),(M) \nsubseteq(V)$ This will also follow from results to be given in Part III.

\section{A method of summation arising from an ALgorithm of Schur}

8. Introduction to Part III. The starting point of Part III is the following theorem of Wall [13].

THEOREM 8.1. Let $e(x)$ be any function analytic and with modulus less than or equal to 1 for $|x|<1$, which is real when $x$ is real. Then there exists a function $F(z)$ of the form

$$
F(z)=\int_{0}^{1} \frac{d \phi(u)}{1+z u}
$$

$\phi(u)$ bounded, monotone non-decreasing, $0 \leqq u \leqq 1$, with modulus less than or equal to 1 for $|z|<1$, such that

$$
\frac{1}{2}(1-x) \frac{1-e(x)}{1+x e(x)}=F(z), \quad z=4 x /(1-x)^{2}, \quad|x|<1 .
$$

Conversely. if $F(z)$ is any function of the form (8.1) with modulus less than or equal to 1 for $|z|<1$, then there exists a function $e(x)$ of the kind described above such that (8.2) holds.

The theorem grows out of an algorithm used by Schur [10] in his work on functions bounded in the unit circle. This algorithm yields a continued fraction representation for the function in the left member of (8.2), and the continued fraction is in turn equal to a function of the form (8.1) with modulus less than or equal to 1 for $|z|<1$. (For details, and a discussion of some consequences of this theorem, see the paper of Wall [13].)

We shall denote by $E$ the class of functions $e(x)$ described in this theorem.

Put $[1-e(x)] /[1+x e(x)]=\sum c_{n}(-x)^{n}, F(z)=\sum c_{n}(-z)^{n}$ in (8.2). Considering the result as a power series identity in $x$, and equating coefficients of like powers of $x$ on either side, we obtain a transformation of the form

$$
C_{n}=\sum_{p=0}^{n} T_{n, p} c_{p}, \quad n=0,1,2, \cdots
$$

From the above theorem it follows that if $e(x) \in E$, then the transformation (8.3) carries the sequence $\left\{c_{p}\right\}$ into a totally monotone sequence $\left\{C_{n}\right\}$. In $\S 9$ we have formulated this in such a way as to characterize a class of functions having positive real part for $|x|<1$.

We find, moreover, that if $\left\{c_{n}\right\}$ is any moment sequence, then $\left\{C_{n}\right\}$ is also a moment sequence, so that $\left[H, C_{n}\right\}$ is a Hausdorff mean. The set of all these means forms a regular manifold in the sense of Part I. 
Considered as a transformation on the series $\sum c_{n}$ to the series $\sum C_{n},(8.3)$ defines a regular method of summation, which we call Schur summability and denote by $(S)$. It turns out that $(S) \approx(M)$, where $(M)$ is the method of Mersman discussed in $\$ 7$. Thus $(S)$ is equivalent to a Gronwall method.

9. Schur summability. If we put $f(x)=\sum c_{n}(-x)^{n}, F(z)=\sum C_{n}(-z)^{n}$ in the relation

$$
(1 / 2)(1-x) f(x)=F(z), \quad z=4 x /(1-x)^{2},
$$

write both members as power series in $x$, and then equate coefficients of like powers of $x$, we obtain the relation

$$
(-1)^{n} c_{n}=\sum_{p=0}^{n}(-1)^{p}(1 / 2)^{2 p+1} C_{n+p, 2 p} C_{p}, \quad n=0,1,2, \cdots
$$

If $(-1)^{n} c_{n}=a_{n},\left(\frac{1}{2}\right)^{2 p+1}(-1)^{p} C_{p}=A_{p}$, this gives

$$
(-1)^{k} A_{k}=t_{k, 0} a_{0}-t_{k, 1} a_{1}+\cdots+(-1)^{k} t_{k, k} a_{k}, \quad k=0,1,2, \cdots,
$$

$t_{k, p}$ being a certain determinant, namely,

$$
t_{k, p}=J_{2 p, k-p}, \quad p=0,1,2, \cdots, k,
$$

where

$$
J_{k, 0}=1, J_{k, m}=\left|\begin{array}{cccccc}
C_{k+1,1}, & C_{k+2,0}, & 0, & 0, & \cdots & 0 \\
C_{k+2,2}, & C_{k+3,1}, & C_{k+4,0}, & 0, & \cdots & 0 \\
\cdot & & . & . & \cdots & 0 \\
\cdot & \cdot & . & . & \cdots & C_{k+2 m-2,0} \\
C_{k+m, m}, & C_{k+m+1, m-1}, & . & . & \cdots & C_{k+2 m-1,1}
\end{array}\right|,
$$

$k=0,1,2, \cdots, m=1,2,3, \cdots$. On subtracting the $(m-k)$ th row from the $(m-k+1)$ th row in succession for $k=1,2,3, \cdots, m-1$, we readily obtain

$$
J_{k, m}=J_{k-1, m}+J_{k+1, m-1}=\sum_{p=1}^{k+1} J_{p, m-1} \text {. }
$$

This holds for $k \geqq 1, m=1,2,3, \cdots$. Hence it follows that

$$
J_{k, m}=(k+1)(k+m+1)^{-1} C_{k+2 m, m}, \quad k \geqq 1, m=0,1,2, \cdots .
$$

This holds also for $k=0$. In fact, if $f(x)=1$ in (9.1), i.e., $c_{0}=1, c_{n}=0, n>0$, then

$$
F(z)=z^{-1}\left[(1+z)^{1 / 2}-1\right]=\sum_{p=0}^{\infty}\left(\frac{1}{2}\right)^{2 p+1}(p+1)^{-1} C_{2 p, p}(-z)^{p},
$$

and consequently $t_{n, 0}=J_{0, n}$ is given by (9.4) with $k=0$. We may now write down at once the inverse of the transformation (9.2): 


$$
C_{n}=\sum_{p=0}^{n} T_{n, p} c_{p}, \quad T_{n, p}=\left(\frac{1}{2}\right)^{2 n+1}(2 p+1)(n+p+1)^{-1} C_{2 n, n-p} .
$$

It is desirable to express this as a transformation on the partial sums $S_{n}=C_{0}+C_{1}+\cdots+C_{n}, s_{n}=c_{1}+c_{0}+\cdots+c_{n}$ of the series $\sum C_{n}, \sum c_{n}$. To do this, let $S_{n}=\sum_{p=0}^{n} b_{n, p} s_{p}, n=0,1,2, \cdots$, and it will be seen that

$$
b_{n, p}=b_{n-1, p}+T_{n, p}-T_{n, p+1},
$$

$p=0,1,2, \cdots, n, b_{n-1, n}=T_{n, n+1}=0$. One may then show by mathematical induction that $b_{n, p}=\left(\frac{1}{2}\right)^{2 n+1} C_{2 n+2, n-p}$. We now make the following definition.

DeFinition. The transformation

$$
S_{n}=\sum_{p m 0}^{n} b_{n, p} s_{p}, \quad b_{n, p}=\left(\frac{1}{2}\right)^{2 n+1} C_{2 n+2, n-p}, \quad n=0,1,2, \cdots,
$$

is called the (S)-transformation; and the method of summation which assigns to the sequence $\left\{s_{n}\right\}$ the value $\lim _{n=\infty} S_{n}$ when the latter limit exists, is called $(S)$ summability.

From Theorem 8.1 and from the way in which $(S)$-summability was defined we have this theorem:

THEOREM 9.1. Let $E$ denote the class of functions $e(x)$ which are analytic and have moduli less than or equal to 1 for $|x|<1$, and which are real when $x$ is real. Then $e(x)$ is in $E$ if and only if $(S)$ transforms the sequence $s_{n}=c_{0}+c_{1}+\cdots+c_{n}$ defined by $[1-e(x)] /[1+x e(x)]=\sum c_{n}(-x)^{n}$ into a sequence $S_{n}=C_{0}+C_{1}$ $+\cdots+C_{n}$, convergent and with limit $\left.{ }^{9}\right)$ less than or equal to 1 , and such that $\left\{C_{n}\right\}$ is a totally monotone sequence.

A theorem equivalent to Theorem 9.1 is as follows:

THEOREM 9.2. Let $K$ denote the class of power series, with real coefficients, of the form

$$
k(x)=\frac{1}{2}-D_{0} x+D_{1} x^{2}-D_{2} x^{3}+\cdots,
$$

convergent and having real part greater than or equal to 0 for $|x|<1$. Then $k(x)$ is in $K$ if and only if $(S)$ transforms the sequence $\left\{1-D_{n}\right\}$ into a sequence $S_{n}=C_{0}+C_{1}+\cdots+C_{n}$ convergent and with limit less than or equal to 1 , and such that $\left\{C_{n}\right\}$ is a totally monotone sequence.

Proof. Let $e(x), k(x)$ be two power series with real coefficients related by the equation $k(x)=1 / 2-x e(x)[1+x e(x)]^{-1}$. Then it is easily seen that $k(x)$ is in $K$ if and only if $e(x)$ is in $E$. Moreover, if $[1-e(x)] /[1+x e(x)]=\sum c_{n}(-x)^{n}$, and $k(x)$ is of the form (9.8), then $1-D_{n}=c_{0}+c_{1}+\cdots+c_{n}, n=0,1,2, \cdots$. The theorem now follows at once from Theorem 9.1. $181]$.

(9) The modulus of $F(z)$ is less than or equal to 1 for $|z|<1$ if and only if $\sum C_{n} \leqq 1[11, \mathrm{p}$. 
10. The $(S)$-transformation and moment sequences. By an application of the work in Part I we shall prove the following theorem.

THEOREM 10.1. The (S)-transformation in the form (9.5) transforms any moment sequence $\left\{c_{n}\right\}$ into another moment sequence $\left\{C_{n}\right\}$ such that $\left[H, C_{n}\right]$ is an essentially regular Hausdorff mean.

Proof. Let $c_{p}=\int_{0}^{1} u^{p} d \phi(u), \phi(u) \in B V[0,1], p=0,1,2, \cdots$. Then by (9.5) we see that

$$
C_{n}=\int_{0}^{1} \beta_{n}(u) d \phi(u), \quad n=0,1,2, \cdots,
$$

where $\beta_{n}(u)=\sum_{p=0}^{n} T_{n, p} u^{p}$. To prove the theorem it is therefore required to show that the sequence $\left\{\beta_{n}(u)\right\}$ is the basis of a regular manifold.

Let $0 \leqq u \leqq 1$. Then $e(x)=u=$ constant is a function in the class $E$ of Theorem 9.1. Also,

$$
[1-e(x)] /[1+x e(x)]=(1-u) /(1+x u)=(1-u) \sum_{p=0}^{\infty} u^{p}(-x)^{p} .
$$

Hence it follows from Theorem 9.1 that the sequence $\left\{(1-u) \beta_{n}(u)\right\}$ is totally monotone, and consequently $\left\{\beta_{n}(u)\right\}$ is totally monotone if $0 \leqq u<1$. It is also totally monotone for $u=1$ by reason of continuity. Therefore $\left\{\beta_{n}(u)\right\}$ is a totally monotone basis of a manifold $M\left[\beta_{n}(u)\right]$.

To show that $M\left[\beta_{n}(u)\right]$ is regular, we put $f(x)=1 /(1+u x)$ in (9.1) and find that the corresponding value of $F(z)$ is

$$
\left[(1-u)+(1+u)(1+z)^{1 / 2}\right]^{-1}=\sum_{p=0}^{\infty} \beta_{p}(u)(-z)^{p}, \quad 0 \leqq u \leqq 1 .
$$

We may write this in the form

$$
\left[(1-u)+(1+u)(1+z)^{1 / 2}\right]^{-1}=\int_{0}^{1} \frac{d_{t} B(u, t)}{1+z t},
$$

where $B(u, t)$ is a monotone function of $t, 0 \leqq t \leqq 1$, for each $u, 0 \leqq u \leqq 1$, determined by the equations $\beta_{p}(u)=\int_{0}^{1} t^{p} d_{t} B(u, t), p=0,1,2, \cdots$. We see by inspection that (10.2) tends to 0 as $z$ tends to $\infty$ through positive real values, in consequence of which $B(u, t)$ is continuous at $t=0$. This in turn implies that $\lim _{n=\infty} \Delta^{n} \beta_{0}(u)=0,0 \leqq u \leqq 1$. Therefore, by Theorem 2.2, $M\left[\beta_{n}(u)\right]$ is regular and the proof of the theorem is complete.

With a view toward finding out other properties of the means in the manifold $M\left[\beta_{n}(u)\right]$ we obtained $B(u, t)$ explicitly from (10.2), using the Stieltjes $[9$, p. 372] inversion formula. The result is

$$
B(u, t)=\frac{1}{\pi} \int_{0}^{t} \frac{1+u}{(1+u)^{2}-4 u s}\left(\frac{1}{s}-1\right)^{1 / 2} d s, \quad 0 \leqq u \leqq 1,0 \leqq t \leqq 1 .
$$


By means of a known $[4$, p. 202] necessary and sufficient condition for a Hausdorff mean to include $(C, n)$ for integral $n$, we find that special means in $M\left[\beta_{n}(u)\right]$ include $(C, 1)$ but not $(C, 2)$. That this is not always the case can be seen from the fact that the regular mean $\left[H, 2 \beta_{n}(1)\right]$, which is in the manifold, does not include $(C, 1)$. In fact, $\beta_{n}(1):(n+1)^{-1} \geqq(n+1) / 4 n^{1 / 2}$, and consequently this ratio cannot be the $n$th member of a moment sequence. Hence

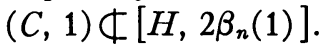

11. The relation of $(S)$-summability to Gronwall summability. On modifying the notation to facilitate comparison with the work in Part II we find that $(S)$-summability is determined by the relation

$$
\sum_{n=0}^{\infty} u_{n}[f(w)]^{n}=\frac{1+(1-w)^{1 / 2}}{(1-w)^{-1}} \sum_{n=0}^{\infty} U_{n} w^{n}, \quad f(w)=\frac{1-(1-w)^{1 / 2}}{1+(1-w)^{1 / 2}},
$$

in the same way that $(f, g)$-summability is determined by (5.1). This is therefore not a Gronwall method inasmuch as the function $1 /(1-w)\left[1+(1-w)^{1 / 2}\right]$ does not satisfy the conditions required by Gronwall for $g(w)$. However, $(S)$ is equivalent to $(M)$, which we showed to be an $(f, g)$-method. In fact, if we put $\left[1+(1-w)^{1 / 2}\right] \sum_{n=0}^{\infty} U_{n} w^{n}=\sum_{n=0}^{\infty} U_{n}^{*} w^{n}$, then (11.1) becomes

$$
\sum_{n=0}^{\infty} u_{n}[f(w)]^{n}=\frac{1}{(1-w)^{-1}} \sum_{n=0}^{\infty} U_{n}^{*} w^{n}
$$

which is formally the same as $(M)$-summability. Now it is easily seen that $\lim _{n=\infty} U_{n}=s$ if and only if $\lim _{n=\infty} U_{n}^{*}=s$, and consequently $(S) \approx(M)$. In fact, if $1+(1-w)^{1 / 2}=\sum v_{n} w^{n}$, then $U_{n}^{*}=v_{0} U_{n}+v_{1} U_{n-1}+\cdots+v_{n} U_{0}$, and since $\sum v_{n}$ converges absolutely and $\sum v_{n}=1$, it follows that $\lim U_{n}=s$ implies $\lim U_{n}^{*}=s$, so that $(S) \subset(M)$. Since $\sum U_{n} w^{n}=w^{-1}\left[1-(1-w)^{1 / 2}\right] \sum U_{n}^{*} w^{n}$, it follows similarly that $(M) \subset(S)$.

Let $S_{n}\left(s_{0}, s_{1}, \cdots\right)$ denote the $n$th $(S)$-sum of the sequence $\left\{s_{n}\right\}$, and $M_{n}\left(s_{0}, s_{1}, \cdots\right)$ the $n$th $(M)$-sum of the same sequence. Then from (9.7), (7.1) it follows that $S_{n}\left(s_{0}, s_{1}, \cdots\right)=(1 / 2) M_{n}\left(s_{0}, s_{1}, \cdots\right)+(1 / 2) M_{n}\left(0, s_{0}, s_{1}, \cdots\right)$. From this and from the fact that $(S) \approx(M)$ we have this theorem:

THEOREM 11.1. If the (S)-limit of the sequence $s_{0}, s_{1}, s_{2}, \cdots$ is $s$, then the $(S)$-limit of the sequence $0, s_{0}, s_{1}, \cdots$ is also $s$.

A number of properties of $(S)$-summability follow from the general theorems about Gronwall summability: e.g., $(V) \subset(S),(S) \nsubseteq(V) ;(S)$ sums the geometrical series in the same domain as that in which $(V)$ sums this series; $(S) \supset(C, k)$ for $k>-1$. In the next paragraph we shall prove some of these things by direct methods.

12. Some properties of $(S)$-summability. Using the relationships obtained in \$\$9-10 we shall prove the following propositions: (a) (S) is regular, (b) (S) sums the geometrical series $\sum x^{n}$ to $1 /(1-x)$ inside the curve 


$$
r=2-\cos \theta+[(1-\cos \theta)(3-\cos \theta)]^{1 / 2},
$$

while $(S)$ does not sum the geometrical series outside or upon this curve; (c) $(V) \subset(S)$; (d) $(S) \nsubseteq(V)$.

Proof of (a). It is required to show that the numbers $b_{n, p}$ of (9.7) satisfy the regularity conditions:

$$
\sum_{p=0}^{n}\left|b_{n, p}\right|<M \text { for every } n, M \text { independent of } n ;
$$

$$
\sum_{p=0}^{n} b_{n, p} \text { tends to } 1 \text { as } n \text { tends to } \infty \text {; }
$$

$$
\lim _{n=\infty} b_{n, p}=0 \text { for every } p \text {. }
$$

From (9.6) we have $\sum_{p=0}^{n} b_{n, p}=\sum_{p=0}^{n} T_{p, 0}$. As $n$ tends to $\infty$ this tends to 1 inasmuch as the power series $\left[(1+z)^{1 / 2}-1\right] / z=\sum_{p=0}^{\infty} T_{n, p}(-z)^{p}$ converges and has the sum 1 when $z=-1$. This proves (ii). Since the $b_{n, p z}$ 's are all positive, (i) holds by virtue of (ii). To prove (iii), we use the relation

$$
b_{n, p}=\sum_{k=p}^{n} T_{k, p}-\sum_{k=p+1}^{n} T_{k, p+1}
$$

which is a consequence of (9.6). As $n \rightarrow \infty$, the two sums of positive terms on the right have finite limits. For if $f(x)=(-x)^{p}$ in (9.1) there results the equation

$$
\left(\frac{(1+z)^{1 / 2}-1}{z}\right)^{2 p+1}=\sum_{k=p}^{\infty} T_{k, p}(-z)^{k-p}
$$

and this series converges for $z=-1$, being the Cauchy product of $2 p+1$ absolutely convergent series. Since the value of the function on the left is 1 when $z=-1$ it follows that $\sum_{k=p}^{\infty} T_{k, p}=1, p=1,2,3, \cdots$. Therefore, by (12.3) we see that the last of the regularity conditions is satisfied, and therefore $(S)$ is regular.

We note for future reference that

$$
\sum_{k=p}^{\infty} T_{k, p} z^{k-p}=(1 / 2)^{2 p+1} F(p+1 / 2, p+1,2 p+2 ; z),
$$

where $F(\alpha, \beta, \gamma ; z)$ is the hypergeometric series.

Proof of (b). On replacing $c_{p}$ by $x^{p}$ in (9.5) we find that in order to show that the geometrical series $\sum x^{p}$ is $(S)$-limitable for a particular value of $x$, it is required to prove that the series $\sum \beta_{p}(x)$ is convergent, where $\beta_{n}(x)$ $=\sum_{p=0}^{n} T_{n, p} x^{p}$. Using the recursion formula (9.3) one may show that these polynomials satisfy the relation. 
(12.6) $\beta_{p}(x)=w\left[\beta_{p-1}(x)-(1+x)^{-1} T_{p-1}(0)\right], w=(1+x)^{2} / 4 x, \beta_{0}(x)=1 / 2$, and consequently $\beta_{n}(x)=(1 / 2) w^{n}-w(1+x)^{-1}\left(T_{0,0} w^{n-1}+T_{1,0} w^{n-2}+\cdots+T_{n-2,0} w+T_{n-1,0}\right)$.

Now the last quantity in parentheses is the coefficient of $t^{n-1}$ in the power series in $t$ for the function $\left[1-(1-t)^{1 / 2}\right] / t(1-w t)$, which is convergent for $t=1$ provided $|w|<1$. It follows that if $|w|<1$,

$$
\sum \beta_{n}(x)=(1 / 2)(1-w)^{-1}-w(1-w)^{-1}(1+x)^{-1}=1 /(1-x) .
$$

On the other hand, if $|w| \geqq 1,|1+x| \geqq 2$, we have

$$
\beta_{n}(x)=w^{n}\left[1 / 2-(1+x)^{-1}\left(T_{0,0}+T_{1,0} w^{-1}+\cdots+T_{n-1,0} w^{1-n}\right)\right] .
$$

Inasmuch as the series $\sum_{p=0}^{\infty} T_{p, 0} w^{-p}$ converges and has a sum numerically less than or equal to 1 it follows that

$$
\left|\beta_{n}(x)\right| \geqq 1 / 2-\left|(1+x)^{-1}\right|, \quad .|w| \geqq 1, \quad|1+x| \geqq 2 .
$$

Now $\sum \beta_{n}(1)$ evidently diverges, being the series for $(1 / 2)(1+z)^{-1 / 2}$ evaluated at $z=-1$. In any other case where $|w| \geqq 1,|1+x| \geqq 2$ we have $\left|\beta_{n}(x)\right| \geqq d>0$, where $d$ is a constant independent of $n$, and hence $\sum \beta_{n}(x)$ diverges. Since the curve $|w|=1,|1+x| \geqq 2$ is given in polar form by (12.1), and since the interior of this curve corresponds to $|w|<1$ and the exterior to $|w|>1$, the proof of (b) is now complete.

Proof of (c). The $n$th $(V)$-sum of the sequence $\left\{s_{n}\right\}$ is given by

$$
V_{n}=\sum_{p=0}^{n} \frac{(n !)^{2}(2 p+1)}{(n-p) !(n+p+1) !} s_{p} .
$$

On eliminating $s_{0}, s_{1}, \cdots, s_{n}$ between (12.7) and (9.7) we obtain a relation of the form $S_{n}=\sum_{p=0}^{n} e_{n, p} V_{p}$, where

$$
\left(\frac{1}{2}\right)^{2 n+1} C_{2 n+2, n-p}=\sum_{k=p}^{n} e_{n, k} \frac{(k !)^{2}(2 p+1)}{(k-p) !(k+p+1) !} .
$$

We shall prove that

$$
e_{n, p}=\left(\frac{1}{2}\right)^{2 n+1} \frac{(2 p) !}{(p !)^{2}} C_{2 n-2 p+1, n-p} .
$$

To do this it will suffice to verify the identity

$$
C_{2 n+2, n-k}=(2 k+1) \sum_{p=k}^{n}(2 p+1)^{-1} C_{2 p+1, p-k} C_{2 n-2 p+1, n-p} .
$$

The quantity on the right may be identified as the coefficient of $x^{n-k}$ in the 
product $F(3 / 2,1,2 ; 4 x) F(k+1 / 2, k+1,2 k+2 ; 4 x)$, while the left member is the coefficient of $x^{n-k}$ in $F(k+3 / 2, k+2,2 k+3 ; 4 x)$. Thus we must establish the power series identity

$F(k+3 / 2, k+2,2 k+3 ; z)=F(3 / 2,1,2 ; z) \cdot F(k+1 / 2, k+1,2 k+2 ; z)$.

Since $D_{z} F(k+1 / 2, k+1,2 k+2 ; z)=(1 / 4)(2 k+1) F(k+3 / 2, k+2,2 k+3 ; z)$, the relation to be established reduces to

$$
F(k+1 / 2, k+1,2 k+2 ; z)=\left(2 \cdot \frac{1-(1-z)^{1 / 2}}{z}\right)^{2 k+1},
$$

which is an identity by (12.4), (12.5).

In order to show that $(V) \subset(S)$, we must show that the regularity conditions (12.2) are satisfied by the numbers $e_{n, p}$. Condition (iii) is clearly satisfied; and (i) will follow from (ii). We have

$$
\sum_{p=0}^{n} e_{n, p}=\left(\frac{1}{2}\right)^{2 n+1} \sum_{p=0}^{n} C_{2 n-2 p+1, n-p} C_{2 p, p} .
$$

This is seen to be the coefficient of $x^{n} 2^{-2 n-1}$ in the power series in $x$ for the function $2\left[1-(1-4 x)^{1 / 2}\right] / 4 x(1-4 x)$. Hence it is required to show that the coefficient of $x^{n}$ in the power series in $x$ for the function $(1-x)^{-1}\left[1-(1-x)^{1 / 2}\right] / x$ has the limit 1 for $n=\infty$. But if this coefficient is denoted by $d_{n}$, we have

$$
d_{n}=v_{0} q_{n}+v_{1} q_{n-1}+\cdots+v_{n} q_{0},
$$

where $q_{0}=q_{1}=\cdots=1$, and $\left[1-(1-x)^{1 / 2}\right] / x=\sum v_{n} x^{n}$. Since $\sum v_{n}$ converges absolutely and $\sum v_{n}=1$, it follows that (12.9) constitutes a regular transformation of the sequence $\left\{q_{n}\right\}$ into the sequence $\left\{d_{n}\right\}$. Hence, inasmuch as $q_{n}=1$ it follows that $\lim d_{n}=1$, as was to be proved. We have completed the proof that $(V) \subset(S)$.

Proof of (d). To show that $(S) \nsubseteq(V)$ we shall show that there is at least one sequence which is summable $(S)$ but which is not summable $(V)$. For that purpose it will suffice to show that if we put $V_{p}=(-1)^{p}$ in the relation $S_{n}=\sum_{p=0}^{n} e_{n, p} V_{p}$ of the preceding proof, then $\lim S_{n}$ exists and is finite. We find that

$$
4^{-1}\left(1-t^{2}\right)^{-1 / 2}\left[(1-t)^{1 / 2}+1\right]=S_{0}+S_{1} t+S_{2} t^{2}+\cdots,
$$

where $S_{n}=\sum_{p=0}^{n}(-1)^{p} e_{n, p}$. Thus if $(1-t)^{1 / 2}+1=\sum v_{p} t^{p}, 4^{-1}\left(1-t^{2}\right)^{-1 / 2}=\sum q_{p} t^{p}$, then $S_{n}=v_{0} q_{n}+v_{1} q_{n-1}+\cdots+v_{n} q_{0}$. This is a regular transformation of the sequence $\left\{q_{n}\right\}$ in to the sequence $\left\{S_{n}\right\}$. Hence, inasmuch as $\lim q_{n}=0$, it follows that $\lim S_{n}=0$. This establishes (d).

\section{BIBLIOGRAPHY}

1. Carlo Birindelli, Sui metodi di Gronwall per la sommazione delle serie, Annali delle Scuòla Normale Superiore di Pisa (2), vol. 8 (1939), pp. 241-270. 
2. - Contributo all'analisi dei metodi di sommazione di Gronwall, Rendiconti del Circolo Matematico di Palermo, vol. 61 (1937), pp. 157-176.

3. H. E. Bray, Elementary properties of Stieltjes integrals, Annals of Mathematics, (2), vol 20 (1918), pp. 177-186.

4. H. L. Garabedian, Einar Hille, and H. S. Wall, Formulations of the Hausdorff inclusion problem, Duke Mathematical Journal, vol. 8 (1941), pp. 193-213.

5. H. L. Garabedian and H. S. Wall, Hausdorff methods of summation and continued fractions, these Transactions, vol. 48 (1940! , pp. 185-207.

6. T. H. Gronwall, Summation of series and conformal mapping, Annals of Mathematics, (2), vol. 33 (1932), pp. 101-117.

7. F. Hausdorff, Summationsmethoden und Momentfolgen. I and II, Mathematische Zeitschrift, vol. 9 (1921), pp. 74-109 and 280-299.

8. W. A. Mersman, A new method of summation of divergent series, Bulletin of the American Mathematical Society, vol. 44 (1938), pp. 667-673.

9. O. Perron, Die Lehre von den Kettenbrüchen, 2d edition, Leipzig and Berlin, 1929.

10. J. Schur, Ueber Potenzreihen die im Innern des Einheitskreises beschränkt sind, Journal für die reine und angewandte Mathematik, vol. 147 (1916), pp. 205-232 and vol. 148 (1917), pp. 122-145.

11. H. S. Wall, Continued fractions and totally monotone sequences, these Transactions, vol. 48 (1940), pp. 165-184.

12. - A class of functions bounded in the unit circle, Duke Mathematical Journal, vol. 7 (1940), pp. 146-153.

13. - Some recent developments in the theory of continued fractions, Bulletin of the American Mathematical Society, vol. 47 (1941), pp. 405-423.

NORTHWESTERN UNIVERSITY,

Evanston, Ill. 